D. Bourzat ${ }^{1}$

P. Souvenir Zafindrajaona $^{2}$

J.J. Lauvergne ${ }^{2}$ V. Zeuh ${ }^{3}$

\title{
Comparaison morpho-biométrique de chèvres au Nord Cameroun et au Tchad
}

BOURZAT (D.), SOUVENIR ZAFINDRAJAONA (P.), LAUVERGNE (J.J.), ZEUH (V.).Comparison morpho-biométrique de chèvres au Nord Cameroun et au Tchad. Revue Elev. Méd. vét. Pays trop., 1993, 46 (4) : 667-674

Deux populations de chèvres femelles adultes du Nord Cameroun et du Tchad (105 animaux au Nord Cameroun et 110 au Tchad) ont été comparées en utilisant une mesure biométrique simple (la hauteur au garrot : HG) et deux mesures combinées (l'indice de gracilité (IGS) = vide sous-sternal/profondeur du thorax et l'indice auriculaire thorax (IAT) = longueur de l'oreille/profondeur du thorax). L'analyse des hauteurs au garrot a permis de distinguer 3 sous-populations, une au Nord Cameroun (de moyenne $m_{1}=\mathbf{5 0 , 2 0} \pm 0,30 \mathrm{~cm}$ ) et deux au Tchad (de moyenne $m_{2}=63,00 \pm 0,60 \mathrm{~cm}$ et $\mathrm{m}_{3}=70,00 \pm 0,40 \mathrm{~cm}$ ). L'indice de gracilité (IGS) était de $1,14 \perp 0,01$ pour la population nord-camerounaise et de $1,48 \pm 0,02$ et $1,56 \pm 0,02$ pour les 2 sous-populations de plus grande taille du Tchad. L'indice auriculaire thorax (IAT) était quasiment identique pour les deux populations tchadiennes (IAT $=0,75 \pm 0,01$ ) alors qu'il était de $0,52 \pm 0,01$ au Nord Cameroun. On peut penser que la sous-population intermédiaire observée au Tchad provient du croisement des 2 populations extrêmes. La distance génétique biométrique de Mahalanobis estimée à partir des trois paramètres (HG, IGS et IAT) fait apparaître que les deux populations du Trhad ne sont pas significativement différentes $\left(D^{2}{ }_{2}=\right.$ 4,30) alors que la population du Nord Cameroun se détache significativement de celles du Tchad $\left(\mathrm{D}_{1-3}^{2}=41,60\right.$ et $\left.\mathrm{D}^{2}{ }_{1-2}=24,40\right)$. L'augmentation linéaire de ces trois paramètres avec lé degré de latitude nord serait due à la pression de sélection naturelle exercée par des conditions de sécheresse et de chaleur de plus en plus sévères lorsque l'on monte vers le nord. Les trois mesures retenues sont facilement calculables, elles sont très discriminantes et peuvent être utilisées avec profit pour la cartographie des ressources génétiques caprines de cette zone de l'Afrique.

Mots-clés: Caprin - Mensuration corporelle - Biométrie - Distance génétique - Cameroun - Tchad.

\section{INTRODUCTION}

On a vu dans un précédent article (8) que deux populations caprines, l'une du Nord Cameroun, l'autre du Tchad peuvent être considérées comme des populations traditionnelles ou primaires au sens donné à ce terme par LAUVERGNE $(6,7)$. En effet, les valeurs prises par les indices de primarité IPs (loci en ségrégation) et IPa (locus Agouti) sont très proches de celles calculées pour des populations du rivage nord de la Méditerranée (8) et

1. CIRAD-EMVT, Laboratoire de recherches vétérinaires et zootechniques (LRVZ) de Farcha, Farcha, BP 433, N'Djamena, Tchad.

2. Département de génétique animale de l'INRA, Laboratoire de Génétique factorielle, bât. 211, CRJ/INRA, 78352 Jouy-en-Josas Cedex, France.

3. Laboratoire de recherches vétérinaires et zootechniques (LRVZ) de Farcha, Farcha, BP 433, N'Djamena, Tchad.

Reçu le 16.7.1993, accepté le 14.09.1993 du Brésil (10). Dans la présente communication, les auteurs se proposent de rechercher une possible discrimination biométrique entre les deux populations caprines de ces deux pays africains en utilisant des mensurations et des indices.

\section{RAPPEL SUR LA CLASSIFICATION DES CHÈVRES D'AFRIQUE}

Les chèvres d'Afrique $(2,5)$ présentent en général des caractéristiques de population primaire, ce qui vient d'être vérifié pour le Nord Cameroun et le Tchad (8). Toutefois, les anciens auteurs ne raisonnaient pas dans cette perspective taxonomique et prenaient seulement en compte des critères biométriques comme la hauteur au garrot et la longueur de l'oreille. Ainsi, pour la chèvre de l'Afrique de l'Ouest une classification en chèvres de grande taille $(70 \mathrm{~cm}$ au garrot), chèvres de taille intermédiaire (65 $\mathrm{cm}$ au garrot) et chèvres de petite taille dites parfois naines (40 à $50 \mathrm{~cm}$ au garrot) a été proposée $(4,11)$. Une telle classification était déjà plus ou moins implicitement admise par des auteurs plus anciens (5).

Par la suite on a voulu prendre aussi en compte la longueur de l'oreille (13) mais, finalement, c'est encore le seul critère de taille que les auteurs les plus récents retiendront pour dresser les premières cartes (à très petite échelle) du peuplement caprin africain $(2,3,17)$.

\section{MATÉRIEL ET MÉTHODES}

\section{Échantillonnage}

L'échantillonnage a été conçu de manière à opérer dans deux zones peuplées d'animaux différant par leur taille: une zone peuplée par de petites chèvres (au Nord Cameroun) et une zone peuplée par des chèvres plus grandes (au Tchad). Ces zones sont délimitées sur la carte de WILSON (17) pour qui la frontière des peuplements petites chèvres/grandes chèvres est délimitée par l'isohyète $750 \mathrm{~mm}$ localisé en gros sur le $11^{\circ}$ de latitude nord (carte 1). La localisation des sites d'échantillonnage (20 troupeaux répartis en 12 sites dans les deux zones Nord Cameroun et Tchad) est donnée sur la carte 1 et les effectifs des animaux examinés dans le tableau l. 


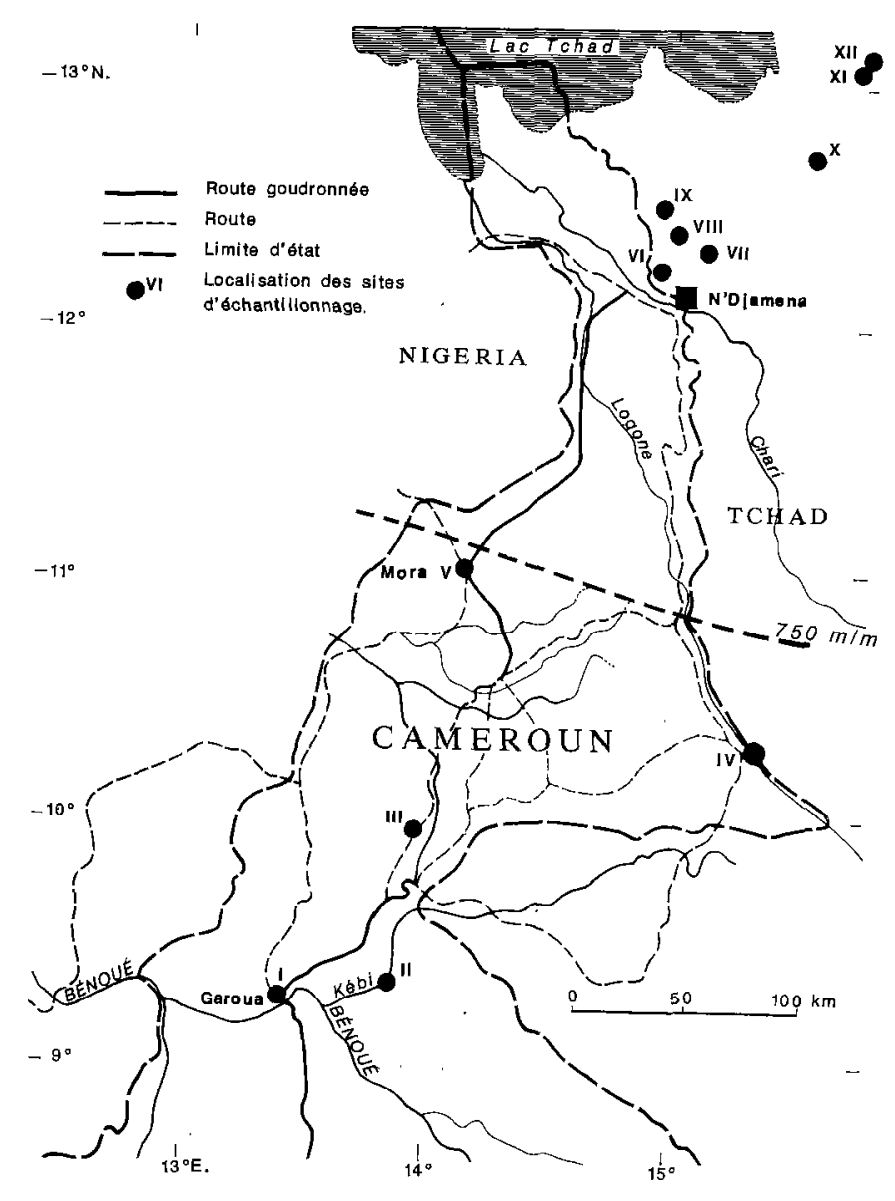

Carte I: Localisation des sites d'échantillonnage.

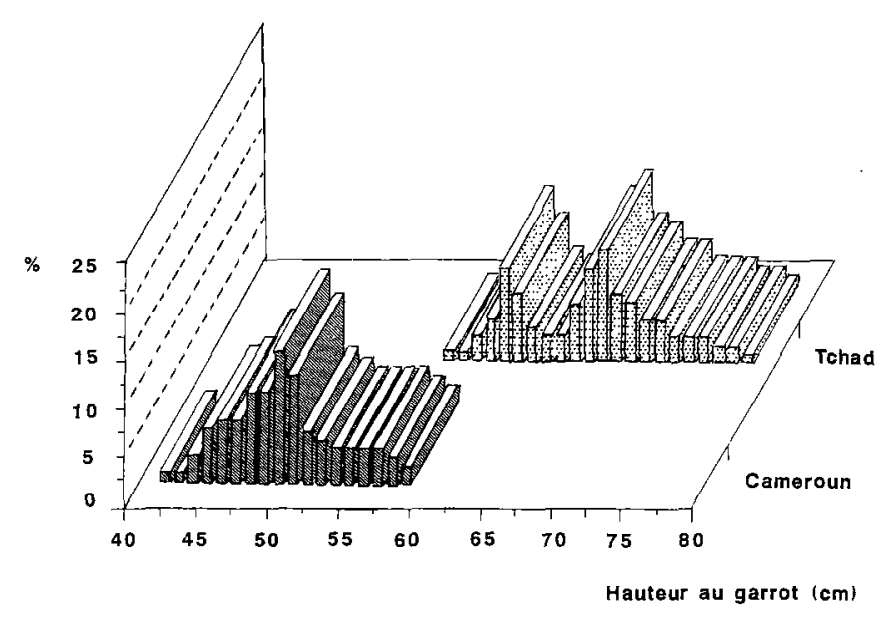

Cameroun Tchad

Figure 1 : Histogramme de la hauteur au garrot (HG) des chèvres du Nord Cameroun et du Tchad.

\section{Les mensurations et leur recueil}

Le protocole général de recueil des données lors de l'enquête caprine de juin 1992 a été donné dans le précédent article (8). Les mensurations intéressant la présente étude sont la longueur de l'oreille de la base jusqu'à la pointe extérieure, la hauteur au garrot et la profondeur du' thorax. Elles ont toutes été prises en centimètre, à l'aide d'un mètre ruban, d'une canne-toise type petits ruminants et d'un compas.

\section{Principe de l'étude}

Pour la présente étude on a retenu 3 paramètres :

- pour la taille, la hauteur au garrot $(\mathrm{HG})$, comme les auteurs précédents (4) ;

- pour l'oreille, un indice auriculaire thorax (IAT) qui rapporte la longueur de l'oreille (LO) à la profondeur du tho$\operatorname{rax}(\mathrm{PT})$ et qui a remplacé la mesure en valeur absolue;

- pour la "hauteur sur patte" - un caractère qui n'a pas encore été pris en considération biométriquement mais qui est remarqué depuis longtemps par les auteurs (4) - a été introduit un indice de gracilité sous-sternal (IGS) : rapport du vide sous-sternal (HG-PT) à la profondeur du thorax (PT).

Le choix de la profondeur du thorax (PT) au dénominateur des deux indices retenus est justifié par sa faible variation d'une population à l'autre. II permet en outre de s'affranchir des variations de longueur des membres. La définition de ces deux indices est donnée dans le tableau II.

\section{Méthodes d'analyse}

Pour discriminer les populations on a tout d'abord analysé un à un les 3 paramètres retenus en regroupant les individus dans les deux zones d'échantillonnage. On a procédé ensuite à une analyse intra-zone et intra-site. On a alors comparé globalement les paramètres biométriques par la distance biométrique de Mahalanobis (9) et l'analyse en composantes principales (15).

Afin d'apprécier l'influence du milieu (climat et végétation) on a rapporté la valeur de ces paramètres au degré de latitude nord qui, dans ces pays, est affecté d'un gradient climatique marqué puisque, en allant du sud au nord on passe du climat soudano-sahélien au climat sahélien (16) avec une réduction continue des précipitations engendrant une raréfaction progressive du couvert végétal donc du fourrage disponible et, par voie de conséquence, une réverbération de plus en plus marquée de la chaleur solaire par le sol dénudé de couleur claire.

\section{RÉSULTATS}

Les histogrammes par zone (Nord Cameroun et Tchad) sont donnés dans les figures 1,2 et 3 pour, respective- 
TABLEAUI Les animaux de l'enquête.

\section{Zone Nord Cameroun}

\begin{tabular}{|c|c|c|c|c|c|}
\hline \multirow{2}{*}{ Site } & \multirow{2}{*}{$\begin{array}{c}\text { Numéro } \\
\text { du troupeau }\end{array}$} & \multirow{2}{*}{ Lieu } & \multirow{2}{*}{ Date } & \multicolumn{2}{|c|}{ Effectifs } \\
\hline & & & & mâles & femelles \\
\hline I. Garoua & $\begin{array}{l}01 \\
02\end{array}$ & $\begin{array}{l}\text { IRZ Garoua } \\
\text { Ouro Orso }\end{array}$ & $\begin{array}{l}2 \text { juin } 1992 \\
2 \text { juin } 1992\end{array}$ & $\begin{array}{l}2 \\
1\end{array}$ & $\begin{array}{l}20 \\
16\end{array}$ \\
\hline II. Bibemi & 03 & Bibemi & 3 juin 1992 & 1 & 8 \\
\hline III. Guider & $\begin{array}{l}04 \\
05\end{array}$ & $\begin{array}{l}\text { Lougguere } 1 \\
\text { Lougguere } 2\end{array}$ & $\begin{array}{l}3 \text { juin } 1992 \\
3 \text { juin } 1992\end{array}$ & $\begin{array}{l}0 \\
0\end{array}$ & $\begin{array}{l}20 \\
11\end{array}$ \\
\hline IV. Yagoua & $\begin{array}{l}06 \\
07 \\
08\end{array}$ & $\begin{array}{l}\text { Kalak } \\
\text { Douroumga } \\
\text { Yagoua }\end{array}$ & $\begin{array}{l}4 \text { juin } 1992 \\
4 \text { juin } 1992 \\
4 \text { juin } 1992\end{array}$ & $\begin{array}{l}0 \\
0 \\
0\end{array}$ & $\begin{array}{r}9 \\
7 \\
10\end{array}$ \\
\hline V. Mora & 09 & Mora & 4 juin 1992 & 1 & 4 \\
\hline Total & & & & 5 & 105 \\
\hline
\end{tabular}

Zone Tchad

\begin{tabular}{|c|c|c|c|c|c|}
\hline \multirow{2}{*}{ Site } & \multirow{2}{*}{$\begin{array}{c}\text { Numéro } \\
\text { du troupeau }\end{array}$} & \multirow{2}{*}{ Lieu } & \multirow{2}{*}{ Date } & \multicolumn{2}{|c|}{ Effectifs } \\
\hline & & & & mâles & femelles \\
\hline VI. Djali & $\begin{array}{l}10 \\
11\end{array}$ & $\begin{array}{l}\text { Djali } 1 \\
\text { Djali } 2\end{array}$ & $\begin{array}{l}8 \text { juin } 1992 \\
8 \text { juin } 1992\end{array}$ & $\begin{array}{l}0 \\
0\end{array}$ & $\begin{array}{l}5 \\
6\end{array}$ \\
\hline VII. Dougui Naga & 12 & Dougui Naga & 9 juin 1992 & 0 & 11 \\
\hline VIII. Am Koundja & 13 & Am Koundja & 9 juin 1992 & 0 & 15 \\
\hline IX. Tom Marefine & $\begin{array}{l}14 \\
15\end{array}$ & $\begin{array}{l}\text { Tom Marefine } 1 \\
\text { Tom Marefine } 2\end{array}$ & $\begin{array}{l}10 \text { juin } 1992 \\
10 \text { juin } 1992\end{array}$ & $\begin{array}{l}0 \\
0\end{array}$ & $\begin{array}{l}20 \\
10\end{array}$ \\
\hline X. Bir Barka & 16 & Bir Barka & 10 juin 1992 & 0 & 13 \\
\hline XI. Dingororoh & $\begin{array}{l}17 \\
18\end{array}$ & $\begin{array}{l}\text { Dingororoh } 1 \\
\text { Dingororoh } 2\end{array}$ & $\begin{array}{l}11 \text { juin } 1992 \\
11 \text { juin } 1992\end{array}$ & $\begin{array}{l}0 \\
0\end{array}$ & $\begin{array}{r}12 \\
5\end{array}$ \\
\hline XII. Lelea & $\begin{array}{l}19 \\
20 \\
\end{array}$ & $\begin{array}{l}\text { Lelea } 1 \\
\text { Lelea } 2 \\
\end{array}$ & $\begin{array}{l}11 \text { juin } 1992 \\
11 \text { juin } 1992\end{array}$ & $\begin{array}{l}0 \\
0\end{array}$ & $\begin{array}{l}5 \\
8\end{array}$ \\
\hline Total & & & & 0 & 110 \\
\hline
\end{tabular}

TABLEAU II Définition des indices biométriques.

\begin{tabular}{|c|c|c|c|}
\hline Appellation & Symbole & Définition & Formule \\
\hline $\begin{array}{l}\text { Indice de } \\
\text { gracilité }\end{array}$ & IGS & $\begin{array}{l}\text { Vide sous-sternal/ } \\
\text { profondeur du thorax }\end{array}$ & (HG-PT)/PT \\
\hline $\begin{array}{l}\text { Indice } \\
\text { auriculaire }\end{array}$ & IAT & $\begin{array}{l}\text { Longueur de l'oreille/ } \\
\text { profondeur du thorax }\end{array}$ & LO/PT \\
\hline
\end{tabular}

TABLEAU III Analyse de variance des paramètres entre zones et entre sites.

\begin{tabular}{|l|c|c|c|}
\hline \multirow{2}{*}{$\begin{array}{c}\text { Source de } \\
\text { variation }\end{array}$} & \multicolumn{3}{|c|}{ Valeur et signification de F } \\
\cline { 2 - 4 } & HG & IGS & \multicolumn{1}{c|}{ IAT } \\
\hline Zones & $\begin{array}{r}715,05 \mathrm{HS} \\
86,40 \mathrm{HS}\end{array}$ & $\begin{array}{r}311,20 \mathrm{HS} \\
33,60 \mathrm{HS}\end{array}$ & $\begin{array}{r}550,20 \mathrm{HS} \\
63,20 \mathrm{HS}\end{array}$ \\
\hline
\end{tabular}


ment, la hauteur au garrot (HG) et les 2 indices (IGS et IAT). L'analyse de variance entre zones et entre sites est donnée dans le tableau III. La variation entre zones est hautement significative, en particulier pour la hauteur au garrot pour laquelle on distingue 3 sous-populations de hauteur au garrot (1 au Nord Cameroun et 2 au Tchad) comme le montre la figure 1 . On a alors isolé les 3 souspopulations identifiées dans l'histogramme des hauteurs au garrot $(\mathrm{HG})$ de la figure 1 et on a comparé les moyennes de leurs indices IGS et IAT (tabl. IV). Dans la figure $4(a, b, c)$, on donne les histogrammes de HG, IGS et IAT par site. Les moyennes des paramètres par site selon le degré de latitude nord sont données dans le tableau $\mathrm{V}$.

Les équations des droites de régression de y (HG, IGS ou IAT) sur $x$, le degré de latitude nord des sites sont (fig. 5) :

$$
\begin{aligned}
& y=5,28 x-0,10 \text { pour HG } \\
& y=0,12 x-0,10 \text { pour IGS } \\
& y=0,07 x-0,22 \text { pour IAT }
\end{aligned}
$$

Dans la figure 6 est donnée la droite de régression de IGS sur IAT. La distance génétique biométrique de Mahalanobis a été calculée à partir d'une combinaison linéaire des trois paramètres (HG, IGS et IAT). II apparaît que les deux sous-populations du Tchad ne sont pas significativement différentes $\left(D^{2}{ }_{2-3}=4,30\right)$ alors que la population du Nord Cameroun se détache significativement de celles du Tchad $\left(D^{2}{ }_{1-3}=41,60\right.$ et $\left.D^{2}{ }_{1-2}=24,40\right)$. Les valeurs des vecteurs propres de l'analyse cn composantes principales sont données dans le tableau VI par paramètre et par sous-population. La représentation graphique est donnée dans la figure $7(a, b, c)$.

\section{DISCUSSION}

Les figures 1,2 et 3 montrent que les 2 grandes zones d'échantillonnage (Nord Cameroun et Tchad) diffèrent significativement pour les 3 paramètres considérés. En outre, pour la hauteur au garrot, il est possible de distinguer deux sous-populations dans la zone tchadienne.

Le tableau IV, dans lequel on a comparé IGS et IAT au sein des 3 sous-populations de hauteurs au garrot, montre que la gracilité varie avec la hauteur au garrot, ce qui est normal si l'on considère que la profondeur du thorax varie très peu entre le Nord Cameroun et le Tchad: tout se joue sur la longueur des pattes.

L'existence au Tchad d'une sous-population intermédiaire pour la taille ( $H G=63 \mathrm{~cm}$ et $I G S=1,48$ ) mais de même indice auriculaire $(I A T=0,75)$ que la population tchadienne de grande taille ( $\mathrm{HG}=70 \mathrm{~cm}$ et $\mathrm{IGS}=1,56)$ s'expliquerait par un croisement entre la grande chèvre du Tchad et la petite chèvre du Nord Cameroun, à condition de supposer que la longueur de l'oreille est déterminée par un variant mendélien dominant et celle de la longueur des pattes par un variant à effet intermédiaire.

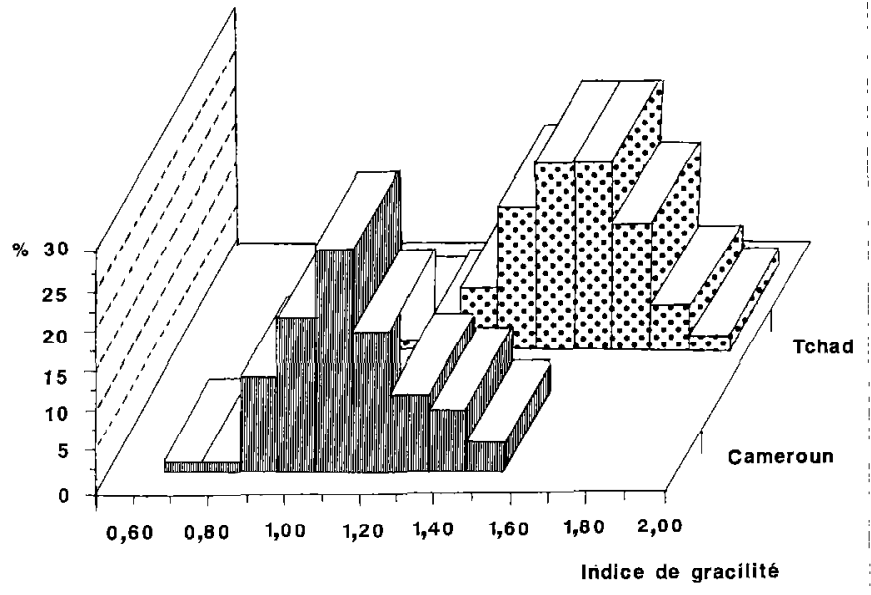

Minlil Cameroun Thehad

Figure 2 : Histogramme de l'indice de gracilité (IGS) des chèvres du. Nord Cameroun et du Tchad.

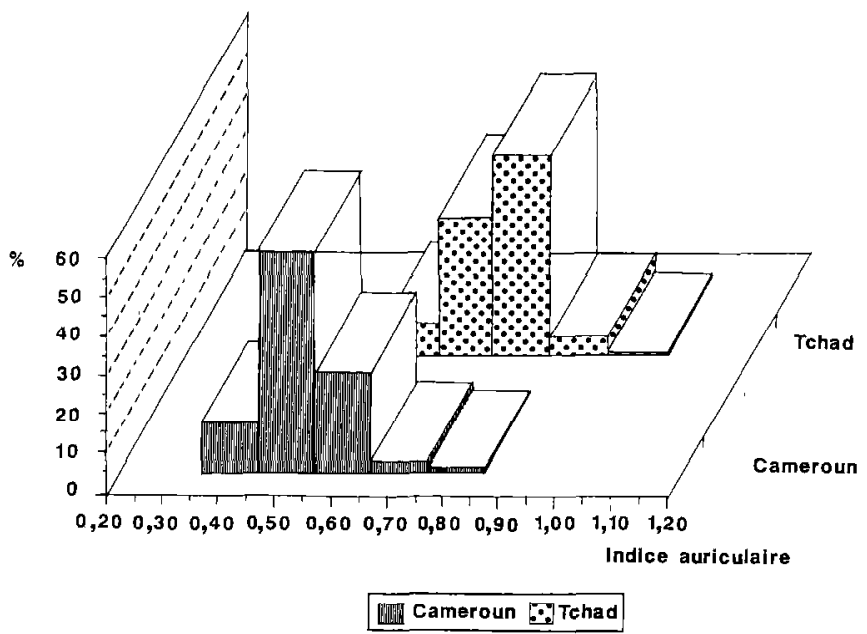

Figure 3 : Histogramme de l'indice auriculaire (IAT) des chèvres du Nord Cameroun et du Tchad.

Cette situation est parfaitement illustrée par la représentation graphique de l'analyse en composantes principales de la figure 7 où seuls la hauteur au garrot $(\mathrm{HG})$ et l'indice de gracité (IGS) permettraient de distinguer les deux sous-populations du Tchad.

La variation de la hauteur au garrot, de l' indice auriculaire et de l'indice de gracilité avec le degré de latitude nord est quasi-linéaire, $\mathrm{HG}$ augmentant en moyenne de $5 \mathrm{~cm}$, et IGS et IAT respectivement de 0,12 et 0,07 point par degré de latitude nord (fig. 5). Si l'on sait que le gradient climatique de cette remontée vers le nord correspond à une diminution de l'humidité qui réduit l'affouragement sur pied disponible, augmentant donc les distances nécessaires pour sa collecte par les animaux, on peut penser qu'alors l'augmentation de la gracilité (allonge- 
TABLEAU IV Comparaison des indices de gracilité et auriculaire entre les deux zones, en distinguant les trois sous-populations de hauteur au garrot.

\begin{tabular}{|c|c|c|c|c|c|c|}
\hline \multirow{3}{*}{$\begin{array}{c}\text { Corps } \\
\text { Symbole }\end{array}$} & \multirow{2}{*}{\multicolumn{2}{|c|}{ 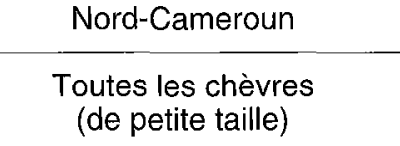 }} & \multicolumn{4}{|c|}{ Tchad } \\
\hline & & & \multicolumn{2}{|c|}{$\begin{array}{l}\text { Chèvre de taille } \\
\text { intermédiaire }\end{array}$} & \multicolumn{2}{|c|}{$\begin{array}{l}\text { Chèvre } \\
\text { de grande taille }\end{array}$} \\
\hline & $n$ & $\mu \pm \sigma$ & $n$ & $\mu \pm \sigma$ & $n$ & $\mu \pm \sigma$ \\
\hline $\begin{array}{l}\text { HG } \\
\text { IGS } \\
\text { IAT }\end{array}$ & $\begin{array}{l}103 \\
103 \\
103\end{array}$ & $\begin{array}{r}50,20 \pm 0,30 \mathrm{a} \\
1,14 \pm 0,01 \mathrm{a} \\
0,52 \pm 0,01 \mathrm{a}\end{array}$ & $\begin{array}{l}33 \\
33 \\
33\end{array}$ & $\begin{array}{r}63,00 \pm 0,60 b \\
1,48 \pm 0,02 b \\
0,75 \pm 0,01 b\end{array}$ & $\begin{array}{l}67 \\
67 \\
67\end{array}$ & $\begin{array}{r}70,00 \pm 0,40 c \\
1,56 \pm 0,02 c \\
0,75 \pm 0,01 b\end{array}$ \\
\hline
\end{tabular}

HG: hauteur au garrot; IGS: indice de gracilité = (hauteur au garrot-profondeur du thorax)/profondeur du thorax ; IAT: indice auriculaire thorax = longueur d'oreille/profondeur de thorax.

Dans un même rang, les moyennes suivies d'une même lettre ne sont pas significativement différentes à $P<0,05$.

TABLEAU V Moyennes de la hauteur au garrot et des indices corporels dans les différents sites suivant le degré de latitude nord.

\begin{tabular}{|c|c|c|c|c|c|}
\hline Pays & Site & $\mathrm{HG}(\mathrm{cm})$ & IGS & IAT & Olatitude \\
\hline Cameroun & $\begin{array}{l}\text { I. Garoua } \\
\text { II. Bibemi } \\
\text { III. Guider } \\
\text { IV. Yagoua } \\
\text { V. Mora }\end{array}$ & $\begin{array}{l}51,25 \\
54,14 \\
47,38 \\
51,27 \\
52,75\end{array}$ & $\begin{array}{l}1,13 \\
1,18 \\
1,10 \\
1,20 \\
1,24\end{array}$ & $\begin{array}{l}0,50 \\
0,54 \\
0,52 \\
0,57 \\
0,60\end{array}$ & $\begin{array}{r}9,30 \\
9,34 \\
9,93 \\
10,30 \\
11,00\end{array}$ \\
\hline Tchad & $\begin{array}{l}\text { VI. Djali } \\
\text { Vil. Dougui-Naga } \\
\text { VIII. Am Koundja } \\
\text { IX. Tom Marefine } \\
\text { X. Bir Barka } \\
\text { XI. Dingororoh } \\
\text { XII. Lelea }\end{array}$ & $\begin{array}{l}68,36 \\
65,54 \\
67,96 \\
67,00 \\
72,12 \\
64,30 \\
65,46\end{array}$ & $\begin{array}{l}1,50 \\
1,45 \\
1,54 \\
1,54 \\
1,65 \\
1,52 \\
1,52\end{array}$ & $\begin{array}{l}0,75 \\
0,70 \\
0,75 \\
0,75 \\
0,80 \\
0,77 \\
0,75\end{array}$ & $\begin{array}{l}12,21 \\
12,28 \\
12,37 \\
12,46 \\
12,63 \\
13,00 \\
13,06\end{array}$ \\
\hline \multicolumn{2}{|c|}{ Intervalle de variations } & $47,38-72,12$ & $1,10-1,65$ & $0,50-0,80$ & $9,30-13,06$ \\
\hline
\end{tabular}

TABLEAU VI Valeur des trois vecteurs propres de l'analyse en composantes principales.

\begin{tabular}{|c|c|c|c|c|}
\hline Paramètres & $\begin{array}{c}\text { Sous- } \\
\text { population }\end{array}$ & Vecteur 1 & Vecteur 2 & Vecteur 3 \\
\hline \multirow{3}{*}{ HG } & 1 & 0,350 & 0,814 & 0,416 \\
& 2 & 0,400 & 0,875 & 0,280 \\
& 3 & 0,450 & 0,876 & 0,170 \\
\hline \multirow{3}{*}{ IGS } & 1 & 0,711 & 0,007 & $-0,703$ \\
& 2 & 0,585 & $-0,072$ & $-0,724$ \\
& 3 & 0,650 & $-0,192$ & $-0,735$ \\
\hline \multirow{2}{*}{ IAT } & 1 & 0,576 & $-0,580$ & 0,576 \\
& 2 & 0,614 & $-0,477$ & 0,628 \\
& 3 & 0,612 & $-0,440$ & 0,655 \\
\hline
\end{tabular}

ment des pattes soutenant un corps de même développement) fait de l'animal gracile un meilleur marcheur et que l'augmentation du vide sous-sternal soustrait dans une certaine mesure l'animal à la réverbération de la chaleur (inversement proportionnelle au carré de la distance) par un sol de plus en dénudé et donc de plus en plus réverbérant lorsque le couvert végétal se raréfie. On a alors un premier élément d'explication du gradient imposé aux indices biométriques par un environnement très contraignant.

L'allongement de l'oreille qui, en gros, multiplie par 4 sa surface, dont on voit l'association étroite avec la gracilité (fig. 6), accompagne apparemment les autres mesures de défense contre la chaleur et la sécheresse en facilitant l'exportation des calories dégagées par le métabolisme par échange thermique de contact (effet de radiateur) plutôt que par exportation de vapeur d'eau dans une zone très sèche (14).

On voit que la comparaison de la taille entre populations n'a de valeur qu'à indice de gracilité égal. Or, dans la littérature cet indice n'a que rarement été calculé. 
D. Bourzat P. Souvenir Zafindrajaona J.J. Lauvergne V. Zeuh
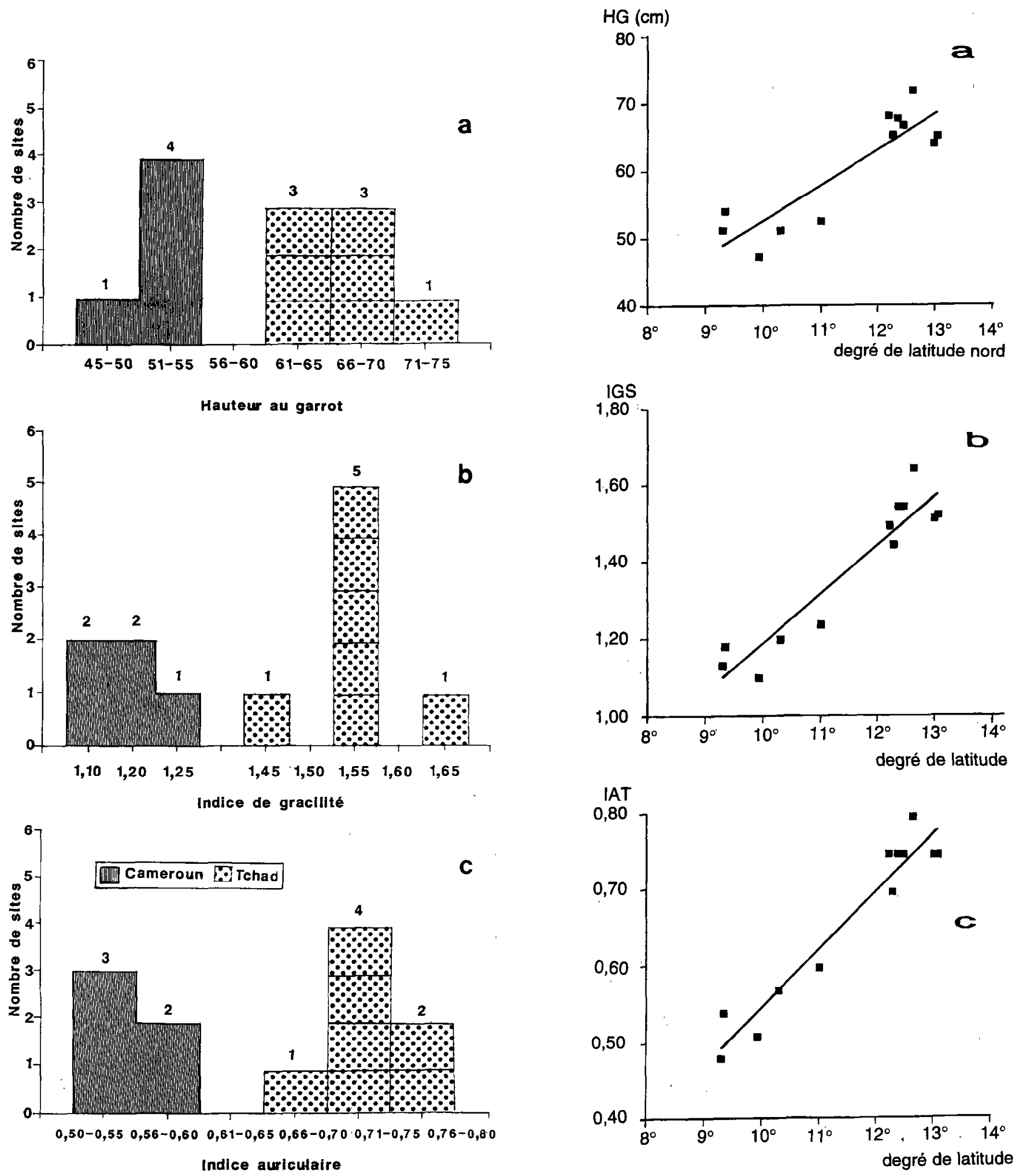

Figure 4: Histogramme des moyennes prises par chaque paramètre dans les sites du Nord Cameroun et du Tchad. a) Hauteur au garrot; b) indice de gracilité ; c) indice auriculaire.

Figure 5: Variation de la hauteur au garrot $(H G)$ (a), de l'indice de gracilité (IGS) (b) et de l'indice auriculaire (IAT) (c) selon le degré de latitude nord. 


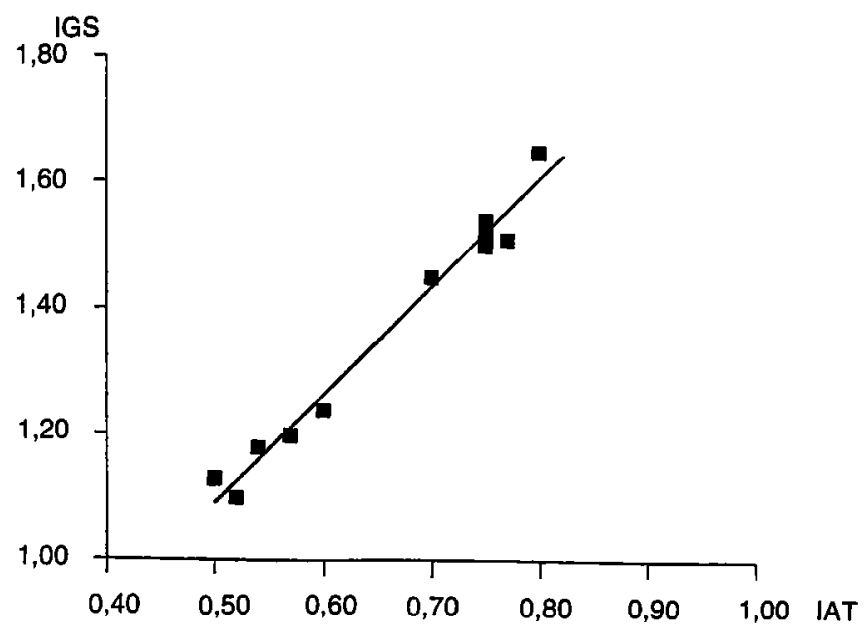

Figure 6 : Équation de la droite de régression de l'indice de gracilité (IGS) sur l'indice auriculaire (IAT).

Toutefois, lors d'un récent sondage sur des chèvres provençales (non publié)les présents auteurs ont trouvé un indice de gracilité moyen de 1,20 (proche de celui des chèvres du Nord Cameroun) pour une hauteur au garrot (HG) de $70 \mathrm{~cm}$. La petite chevre africaine serait en quelque sorte homologue dans ses proportions de la chèvre européenne dans un rapport de 0,70 . Les grandes chèvres de l'Inde avec des hauteurs au garrot de 70 à $85 \mathrm{~cm}$ (12) n'atteignent apparemment cette taille que par augmentation de la longueur de leurs pattes. Comme ordre de grandeur de l'indice de gracilité pour ce type d'animal on a en effet comme au Tchad des valeurs de 1,50 pour la femelle Kutchi $(82,4 \mathrm{~cm}$ au garrot) et de 1,57 pour la femelle Jhakrana $(79,2 \mathrm{~cm}$ au garrot ) (valeurs calculées d'après (1)). Ces observations confortent donc celles trouvées lors de cette enquête.

\section{CONCLUSION}

Cette étude porte les bases d'une cartographie simple des ressources génétiques caprines africaines, au moins dans les zones sahélienne et soudano-sahélienne. Cette cartographie pourrait se faire en trois temps :

1. mesure des indices de primarité définis en (8), de manière à vérifier l'état généralisé de la primarité ;

2. mesure de la hauteur au garrot, de l'indice auriculaire et de l'indice de gracilité selon un échantillonnage tenant compte de la densité de peuplement ;

3. représentation cartographique de ces paramètres pris d'abord individuellement pour isoler les zones par des iso-HG, -IGS ou -IAT qui permettront de déterminer dans un second temps les principales zones ethniques.
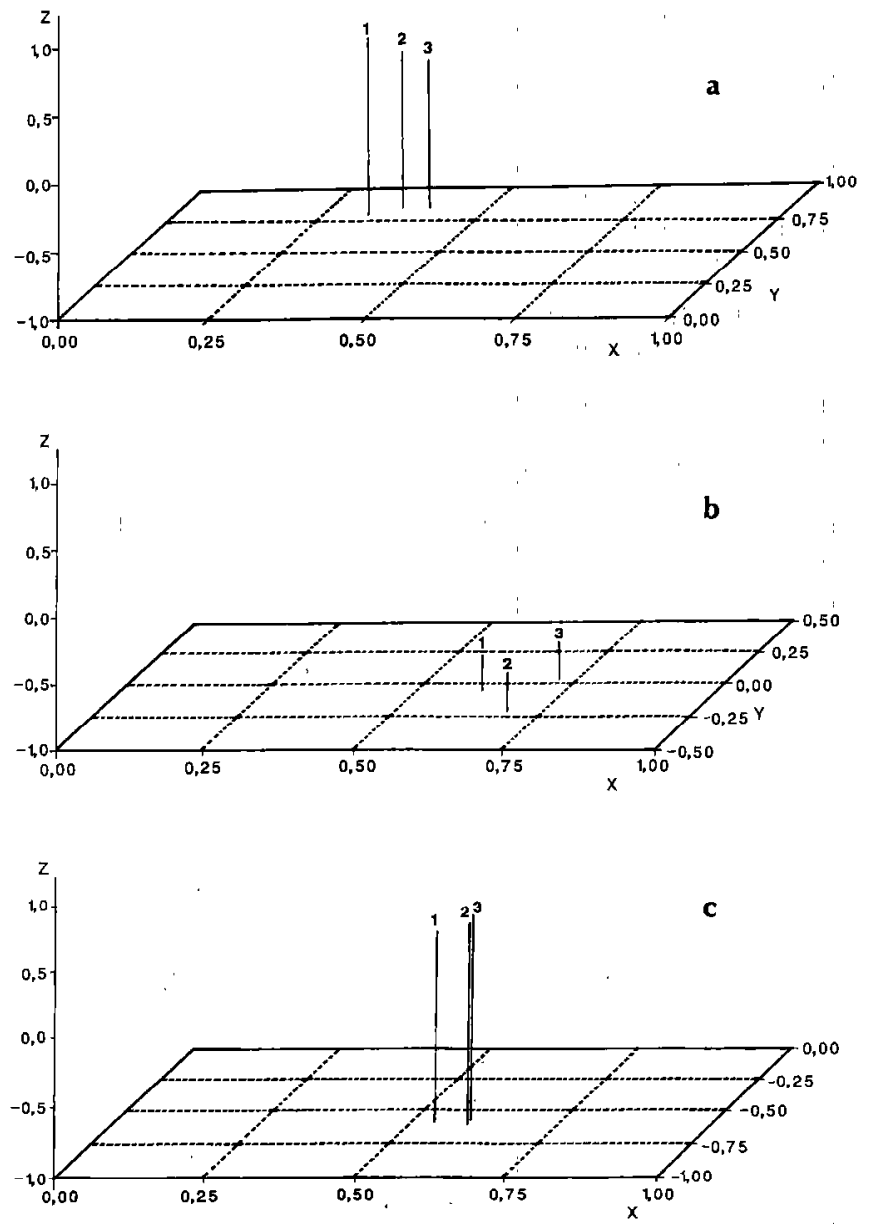

Figure 7 : Représentation graphique de l'analyse en composantes principales pour la hauteur au garrot $(H G)(a)$, l'indice de gracilité (IGS) (b) et l'indice auriculaire (IAT) (c).

\section{BIBLIOGRAPHIE}

1. ACHARYA (R.M.). Sheep and Goat Breeds of India. Rome, FAO, 1982. 190 p. (Animal Production and Health Paper $N^{\circ} 30$ )

2. DAPPER (O.), TEN RHYNE (W.), GREVENBROCK (J.C. de). The Early Cape Hottentots (1668). Translated by SHAPOERA and FARRINGTON, 1933 (cité par EPSTEIN (5))

3. DEVENDRA (C.), BURNS MARCA. Goat Production in the Tropics. 2nd ed. Farnham Royal. CAB, 1983. (CAB Techn. Comm. N¹9)

4. DOUTRESSOULE (G.). L'élevage en Afrique Occidentale Française. Paris. Larose, 1947. 298 p.

5. EPSTEIN (H.). The Origin of the Domestic Animals of Africa. Vol. 2. New York, London, Munich, Africana, 1971. P. 195-209 ; 210-235;237$295 ; 296-305 ; 307-309$.

6. LAUVERGNE (J.J.). Genética en poblaciones animales después de la domesticación : concecuencias para la conservación de las razas. In : Proceedings of the 2nd World Congress on Genetics applied to Livestock Production, Madrid (Spain), 4-8 October 1982. Vol. 6. p. 77-87. 


\section{Bourzat P. Souvenir Zafindrajaona I.J. Lauvergne V. Zeuh}

7. LAUVERGNE (J.J.). Breed Development and Breed Differenciation. In : Proc. CEC Workshop and Training Course, Hannover, Dec. 7-9 1992 Brıssels, Commission of the European Communities, Agriculture. p. 5364.

8. LAUVERGNE (JJ) BOURZAT (D) SOUVENIR ZAFINDRAJAONA (P.), ZEUH (V.), NGO TAMA (A.-C.). Indices de primarité de chèvres au Nord Cameroun et au Tchad. Revue Élev. Méd. vét. Pays trop., 1993, 46 (4) : 651-665.

9. LEFORT-BUSON (M.), VIENNE (D. de). Les distances génétiques (estimations et applications). Paris, INRA, 1985. p. 23-57.

10. MACHADO (T.), LAUVERGNE (J.J.), SOUVENIR ZAFINDRAJAONA (P.). Le scénario de peuplement caprin brésilien depuis la découverte. Arch. Zootec. Cordoba, 1992, $41: 455-466$.

11. MASON (I.L.). The Classification of West African Livestock. Farnham Royal, Bucks, CAB, 1951. p. 33-34.

12. MASON (I.L.). 3. Breeds .In : GALL (C.). Goat Production. London, Academic Press, 1981. p. 57-109.

BOURZAT (D.), SOUVENIR ZAFINDRAJAONA (P.), LAUVERGNE (J.J.), ZEUH (V.) A morpho-biometric comparison between goats from Northern Cameroon and Chad. Revue Élev. Méd. vét. Pays trop., 1993, 46 (4) : 667-674

Two populations of adult female goats from Northern Cameroon and Chad (105 and 110 animals, respectively) were compared using a simple biometric measure (wither height : HG) and two combined measures $($ the index of slenderness $($ IGS $)=$ volume of sub-sternal cavity/thorax depth and the auricle-thorax index (IAT) $=$ auricle lenglh/thurax dep(h). The analysis of wither heights revealed 3 subpopulations : one in Northern Cameroon (mean number $\mathrm{m}_{1}=\mathbf{5 0 . 2 0}$ $\pm 0.30 \mathrm{~cm}$ ) and two in Chad (mean number $m_{2}=63.00 \pm 0.60 \mathrm{~cm}$ and $m_{1}=70.00 \pm 0.40 \mathrm{~cm}$ ). The index of slenderness (IGS) reached $1.14 \pm$ 0.01 for the population from Northern Cameroon and $1.48 \pm \mathbf{0 . 0 2}$ and $\mathbf{1 . 5 6} \pm \mathbf{0 . 0 2}$ for the two largest Chadian sub-populations. The auricle-thorax index (IAT) remained almost unchanged in both Chadian populations $(\mathrm{IAT}=\mathbf{0 . 7 5} \pm \mathbf{0 . 0 1}$ ), but reached $0.52 \pm 0.01$ in Northern Cameroon. It may be assumed that the intermediate sub-population observed in Chad resulted from the crossing between the two extreme populations. The biometric genetic distance of Mahalanobis based on the three parameters (HG, IGS and IAT) showed a non significant difference between the two Chadian populations $\left(\mathrm{D}^{2}{ }_{3}=\right.$ 4.30). In contrast, the differences between the population from Northern Cameroon and Chad were significant $\left(\mathrm{D}_{1-3}^{2}=41.60\right.$ and $\mathrm{D}^{2}{ }_{-2}=$ 24.40). The linear increase in these three parameters with the Northern degree of latitude could be due to the pressure of natural selection resulting from the ever more severe drought and heat conditions encountered further North. The three measures can be easily calculated, they are discriminating and can be effectively used for the mapping of caprine genetic resources at least in this area of Africa.

Key words : Goat - Body measurement - Biometry - Genetic distance Cameroon - Chad.
13. MASON (I.L.), MAULE (J.P.). The Indigenous Livestock of Eastern and Southern Africa. Farnham Royal, CAB, 1960. 240 p. (Technical Communication $\mathrm{N}^{\circ} 14$ )

14. ROBERTSHAW (D.). Concepts in Animal Adaptation, Thermoregulation of the Goat. $I n$ : Proc. Int. Conf., Goat, Production and Disease, Tucson, Arizona,1982. Scottsdale, Arizona, USA, Dairy Goat Publishing Co., 1982. p. 395-397.

15. TOMASSONE (R.). Biométrie (mathématique et informatique). Paris, Institut National Agronomique Paris-Grignon, 1987. p. 24-42.

16. TRAIL (J.C.M.), LHOSTE (C.H.), VISSOCQ (Y.J.), LHOSTE (Ph.), MASON (I.L.). Trypanotolerant Livestock in West and Central Africa. 2 Vol. General study and Country studies. Addis Ababa, ILCA, 1979. 147p ; 303 p.

17. WILSON (R.T.). Small ruminants production and the small ruminants genetic resources in tropical Africa. Rome, FAO, 1991. 231 p. (Animal Production Health Paper $\mathrm{N}^{\circ} 88$ )

BOURZAT (D.), SOUVENIR ZAFINDRAJAONA (P.), LAU VERGNE (J.J.), ZEUH (V.) Comparación morfo-biométrica de cabras en el norte de Camerún y en Chad. Revue Élev. Méd. vét. Pays trop., 1993,46 (4) : 667-674

Se compararon dos poblaciones de cabras, todas hembras adultas, del norte de Camerún y de Chad (105 animales en el norte de Camerún y 110 en Chad), gracias a una medida biométrica simple (alzada a la cruz : HG) y a dos medidas combinadas (índice de gracilidad (IGS) = vacío sub-esternal/profundidad torácica e índice auricular torácico (IA'I') = longitud de la oreja/profundidad torácica). El análisis de la alzada a la cruz permite distinguir 3 sub-poblaciones, una al norte de Camerún (con un promedio $\mathrm{m}_{\mathbf{1}}=\mathbf{5 0 , 2 0} \pm \mathbf{0 , 3 0} \mathrm{cm}$ ) y dos en Chad (con promedios de $m_{2}=63,00 \pm 0,60 \mathrm{~cm}$ y $\mathrm{m}_{3}=70,00 \pm 0,40 \mathrm{~cm}$ ). El indice de gracilidad (IGS) fue de $1,14 \pm 0,01$ para la población del norte de Camerún y de $1,48 \pm 0,02$ y de $1,56 \pm 0,02$ para las dos poblaciones mayores de Chad. El índice auricular torácico (IAT) fue casi idéntico para las dos poblaciones chadianas ( $\mathrm{IAT}=0,75 \pm 0,01$ ), mientras que para la población nor-camerunesa fue de $0,52 \pm 0,01$. Se sugiere que la sub-población chadiana, intermedia, podría provenir de un cruce entre las dos poblaciones extremas. La distancia biométrica genética de Mahalanobis, estimada a partir de los tres parámetros (HG, IGS y IAT), muestra que las dos poblaciones de Chad no difieren en forma significativa $\left(D^{2}=4,30\right)$, mientras que la población al norte de Camerún difiere significativamente de las chadianas $\left(\mathrm{D}^{2}{ }_{1-3}=41,60 \mathrm{y}\right.$ $D^{2}{ }_{1,2}=24,40$ ). El aumento linear de estos tres parámetros con respecto al grado de latitud norte, podría deberse a la presión natural que ejercen las condiciones de sequía y de calor, las cuáles son más severas al dirigirse hacia el norte. Las tres medidas se calculan facilmente, son discriminantes y pueden utilizarse para la cartografía de los recursos genéticos caprinos, al menos en esta zona de Africa.

Palabras claves : Caprino - Medida corporal - Biometría - Distancia genética - Camerún - Chad. 